\title{
On a fourth-order envelope equation for deep-water waves
}

\author{
By PETER A. E. M. JANSSEN $\dagger$ \\ Applied Mathematics, California Institute of Technology, Pasadena, CA 91125
}

(Received 4 March 1982)

The ordinary nonlinear Schrödinger equation for deep-water waves (found by a perturbation analysis to $O\left(\epsilon^{3}\right)$ in the wave steepness $\epsilon$ ) compares unfavourably with the exact calculations of Longuet-Higgins (1978) for $\epsilon>0 \cdot 10$. Dysthe (1979) showed that a significant improvement is found by taking the perturbation analysis one step further to $O\left(\epsilon^{4}\right)$. One of the dominant new effects is the wave-induced mean flow. We elaborate the Dysthe approach by investigating the effect of the wave-induced flow on the long-time behaviour of the Benjamin-Feir instability. The occurrence of a wave-induced flow may give rise to a Doppler shift in the frequency of the carrier wave and therefore could explain the observed down-shift in experiment (Lake et al. 1977). However, we present arguments why this is not a proper explanation. Finally, we apply the Dysthe equations to a homogeneous random field of gravity waves and obtain the nonlinear energy-transfer function recently found by Dungey \& Hui (1979).

\section{Introduction}

It is well-known that a finite-amplitude uniform train of surface gravity waves is unstable to modulational perturbations with sufficiently long wavelength (BenjaminFeir instability). Benjamin \& Feir (1967) obtained a fair agreement between the experimentally determined growth rates of the sidebands and theory, although the experimental values were systematically below the theoretical curve (henceforth to be called the Benjamin-Feir growth-rate curve). This theoretical growth-rate curve is most easily determined from the nonlinear Schrödinger equation, which is the evolution equation for the slowly varying envelope of the carrier wave. We remark that the nonlinear Schrödinger equation has only a restricted validity. It assumes that the spatial variations of the envelope are of the same order as the wave steepness of the carrier wave. Therefore the Benjamin-Feir growth-rate curve also has a restricted validity. This was pointed out by Longuet-Higgins (1978), who solved the exact equations for surface gravity waves by means of the computer. The agreement of his theoretical results with experiments of Benjamin \& Feir (1967) and Lake et al. (1977) is even better. Similar results were obtained by Crawford et al. (1981) from the so-called Zakharov equations. On the other hand, Dysthe (1979) gave an improved version of the nonlinear Schrödinger equation by including higher-order effects. Surprisingly, his analysis showed that up to a wave steepness of $0 \cdot 25$ the effect of the modulation-induced current could explain the difference between the approximate results of Benjamin \& Feir and the exact result of Longuet-Higgins. The Dysthe equations therefore seem to give an appropriate description of the dynamical behaviour of e.g. sea waves, since for those waves the wave steepness is small enough.

† Permanent address: K.N.M.I, De Bilt, The Netherlands. 
In this paper we intend to elaborate the Dysthe approach. In $\S 2$ we present a coupled set of equations for the envelope of the carrier wave and the wave-induced mean flow. We solve for the wave-induced mean flow to obtain a single evolution equation for the envelope of the wave (to be called the fourth-order envelope equation). We intend to study some interesting properties of the fourth-order envelope equation. Firstly, we review the linear stability analysis of a uniform wavetrain, as given by Dysthe (1979), and we give additional evidence to support Dysthe's conclusion that the higher-order effect of the wave-induced flow yields a surprising improvement of the theoretical results $(\$ 2)$. Secondly, in $\$ 3$ we study the long-time behaviour of a single unstable mode near the threshold for instability. The long-time behaviour of this instability shows recurrence, qualitatively in agreement with experiments of Lake et al. (1977). For the latter experiment also a shift in the frequency of the carrier wave was found. The occurrence of a wave-induced current may give rise to a Doppler shift in the frequency shift and therefore could explain the observed shift in experiment. However, we show from the analysis that in the framework of the fourth-order envelope equation the frequency shift is periodic in space and time, in contrast with observation (Lake et al. 1977). Therefore, other effects, such as dissipation, must be responsible for the observed frequency shift. Finally, in $\$ 4$ we briefly study the problem of energy transfer due to resonant four-wave interactions for a random homogeneous field of gravity waves. Longuet-Higgins (1976) considered this problem for the ordinary nonlinear Schrödinger equation and found that the transfer function is just a constant. From the fourth-order envelope equation one would therefore expect corrections to the transfer function found by Longuet-Higgins, which are proportional to the spectral width. And indeed, the transfer function we obtain from the fourth-order envelope equation is just the one given by Dungey \& Hui (1979). These authors showed that the effect of spectral width on the nonlinear energy transfer is far from negligible. Hence, also for a random wavefield the higher-order effects in the fourth-order envelope equation are surprisingly important. Section 5 summarizes our conclusions.

\section{The evolution equations and linear theory}

In this section we present the fourth-order envelope equation for water waves in a fluid of infinite depth. We are interested in the evolution of a narrow-band spectrum with small wave steepness so that in first order the velocity potential $\phi$ is given by

$$
\phi \approx\left(A e^{i \theta}+\text { c.c. }\right) e^{k_{0} z}, \quad \theta=k_{0} x-\omega_{0} t,
$$

where $\omega_{0}$ and $k_{0}$ are the frequency and wavenumber of the carrier wave $\left(\omega_{0}^{2}=g k_{0}\right)$, and c.c. means complex conjugate. We note that the carrier wave propagates in the $x$ direction; $g$ is the acceleration due to gravity, which is pointed in the negative $z$-direction. The complex amplitude $A$ is slowly varying in time and space $(x, y, z)$. For deep water, Zakharov (1968) obtained the nonlinear Schrödinger equation as the evolution equation for the complex amplitude $A$. Dysthe (1979) extended this to include higher-order effects. Using the method of multiple scales, he obtained a coupled set of evolution equations for the envelope $A$ of the carrier wave and the wave-induced mean flow (with potential $\bar{\phi}$ ). (See equations $(2.19)$ and $(2.21)$ of Dysthe's paper. Note that the third term on the right-hand side of $(2.19)$ should have a minus sign.) One can easily solve the equations for the potential $\bar{\phi}$ of the mean flow $\bar{u}$ by means of the Fourier-transform technique to arrive at a single equation for the envelope of the wave (henceforth to be called the fourth-order envelope equation). 
Introducing dimensionless units, $t^{\prime}=\omega_{0} t, \mathbf{x}^{\prime}=k_{0} \mathbf{x}, A^{\prime}=2 k_{0}^{2} A / \omega_{0}$, and dropping the primes, the equation for the dimensionless envelope $A$ (which, for a uniform wavetrain, is now just the constant wave steepness) reads

$$
\begin{aligned}
& 2 i\left(\frac{\partial}{\partial t} A+\frac{1}{2} \frac{\partial}{\partial x} A\right)-\frac{1}{4} \frac{\partial^{2}}{\partial x^{2}} A+\frac{1}{2} \frac{\partial^{2}}{\partial y^{2}} A-|A|^{2} A \\
& \quad=\frac{i}{8}\left(\frac{\partial^{3}}{\partial x^{3}}-6 \frac{\partial^{3}}{\partial x \partial y^{2}}\right) A+\frac{1}{2} i A\left(A \frac{\partial}{\partial x} A^{*}-A^{*} \frac{\partial}{\partial x} A\right)-\frac{5}{2} i|A|^{2} \frac{\partial}{\partial x} A+A \mathscr{H} \frac{\partial}{\partial x}|A|^{2},
\end{aligned}
$$

where the two-dimensional version of the Hilbert transform $\mathscr{H}$ reads

$$
\mathscr{H} \psi=\frac{1}{2 \pi} \int d \mathbf{x}^{\prime} \frac{x^{\prime}-x}{r^{3}} \psi\left(\mathbf{x}^{\prime}\right), \quad r=\left[\left(x^{\prime}-x\right)^{2}+\left(y^{\prime}-y\right)^{2}\right]^{\frac{1}{2}} .
$$

In deriving (2) it was assumed that $A=O(\epsilon)$ and a derivative increases the order by one. The ordinary nonlinear Schrödinger equation is obtained from (2) by neglecting its right-hand side, which is of order $\epsilon^{4}$. The small wave-induced current, which is caused by the radiation stresses of the modulated wavetrain (Longuet-Higgins \& Stewart 1964), is among others represented by the term involving the Hilbert transform $\mathscr{H}$. In the remaining part of this paper we intend to study some properties of (4). To that end we first review the linear stability of a uniform wavetrain (Dysthe 1979). Of special interest is the effect of the wave-induced current.

Dysthe studied the linear stability of a finite-amplitude uniform wavetrain to two-dimensional perturbations and compared the results with numerical calculations of Longuet-Higgins (1978). The agreement was surprisingly good for a wave steepness $A<0 \cdot 25$.

We, however, consider the stability of a uniform wavetrain to one-dimensional perturbations only. Since Dysthe showed that only the term involving the Hilbert transform contributes to the stability results, we start in the one-dimensional case with

$$
2 i \frac{\partial}{\partial t} A-\frac{1}{4} \frac{\partial^{2}}{\partial x^{2}} A=\left(|A|^{2}-A_{0}^{2}\right) A+A \mathscr{H}^{(1)} \frac{\partial}{\partial x}|A|^{2},
$$

where we have transformed to a frame moving with the group velocity and the one-dimensional version of the Hilbert transform is given by

$$
\mathscr{H}^{(\mathbf{1})} \psi=\frac{1}{\pi} \mathscr{P} \int \frac{d x^{\prime}}{x^{\prime}-x} \psi\left(x^{\prime}\right)
$$

Here the symbol $\mathscr{P}$ stands for principal value. In addition, to obtain (4) from (2) we have used $\tilde{A}=A \exp \left(+i A_{0}^{2} t\right)$ (where $A_{0}$ is a constant) and have dropped the tilde. A steady-state solution of (5) is $A=A_{0}$ and to study its stability we perturb it according to

$$
A=A_{0}+A_{1}, \quad A_{1} \ll A_{0} .
$$

Linearizing around the steady state, we obtain for normal modes of the form $A_{1} \sim \exp i(k x+\omega t)$ the dispersion relation

$$
\omega^{2}=\frac{1}{8} k^{2}\left(\frac{1}{8} k^{2}-A_{0}^{2}(1-|k|)\right) .
$$

The effect of the wave-induced current is given by the modulus-of- $k$ term. $\dagger$ From (7) we infer at once that we have instability of the uniform wavetrain $\left(\omega^{2}<0\right)$ if the wave steepness $A_{0}$ is sufficiently large (modulational instability), i.e.

$\dagger$ The neglected terms only give contributions of the order of $A_{0}^{4}$ to the expression of $\omega^{2}$. 


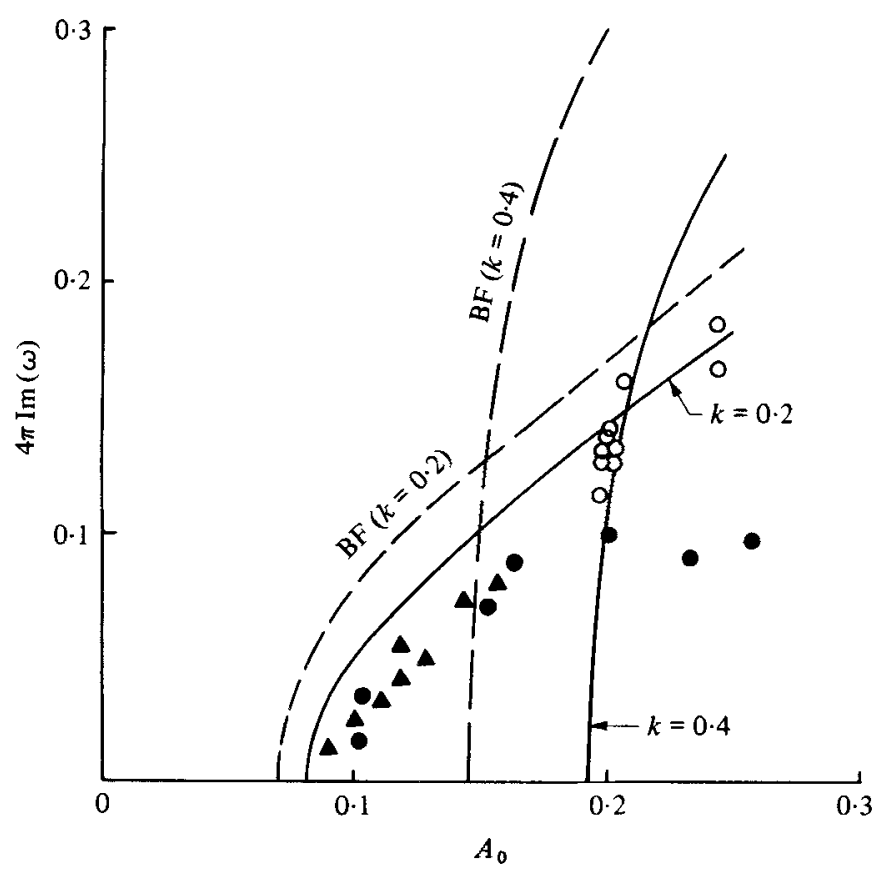

Figure 1. The normalized sideband growth rate $4 \pi \operatorname{Im}(\omega)$ as a function of wave steepness $A_{0}$ for two values of modulation wavenumber $k$. - , (7); ---, theoretical result of Benjamin \& Feir. Experimental results: $\bigcirc, k=0 \cdot 4$, Lake et al. (1977);,$k=0 \cdot 2$, Lake et al. (1977); $\mathbf{\Delta}, k=0 \cdot 2$, Benjamin (1967).

$A_{0}^{2}>\frac{1}{8} k^{2} /(1-|k|)$. This feature is illustrated in figure 1 , where we have plotted the normalized sideband growth rate $4 \pi \operatorname{Im}(\omega)$ versus the wave steepness $A_{0}$ for two values of $k$. To see the effect of the wave-induced current more clearly we have plotted for comparison the growth-rate curve of the ordinary nonlinear Schrödinger equation (i.e. (7) without the modulus of $k$ term). Also shown are experimental results of Benjamin (1967) and Lake et al. (1977). We conclude that inclusion of the effect of wave-induced current indeed gives an improvement, especially for $k=0 \cdot 4$. Figure 2 gives the dimensionless growth rate $\operatorname{Im}(\omega) / \frac{1}{2} A_{0}^{2}$ as a function of the normalized wavenumber $k / 2 A_{0}$ for various values of the wave steepness $A_{0}$. The curve with $A_{0}=0$ corresponds to the ordinary nonlinear Schrödinger result. It is remarkable that even for $A_{0}=0.1$ the effect of the wave-induced current is considerable. For comparison, we have also shown in figure 2 results of Crawford et al. (1981). As a starting point for the stability analysis of a uniform wavetrain, these authors used the Zakharov equation for weakly nonlinear water waves, an equation which retains all the higher-order dispersion effects, but which is correct to third order in amplitude only. The results from the fourth-order envelope equation seem to agree with those of the Zakharov equation for a wave steepness $A_{0}<0 \cdot 2-0 \cdot 25$.

To summarize the results of the stability of a uniform wavetrain in one dimension we conclude that the addition of the effect of the wave-induced current to the nonlinear Schrödinger equation gives a surprising improvement. Thus, as far as stability properties are concerned it is sufficient to use the simplified version of the modified nonlinear Schrödinger equation, namely (4). In $\S 3$ we use (4) to study the nonlinear stability of the finite-amplitude, uniform wavetrain. 


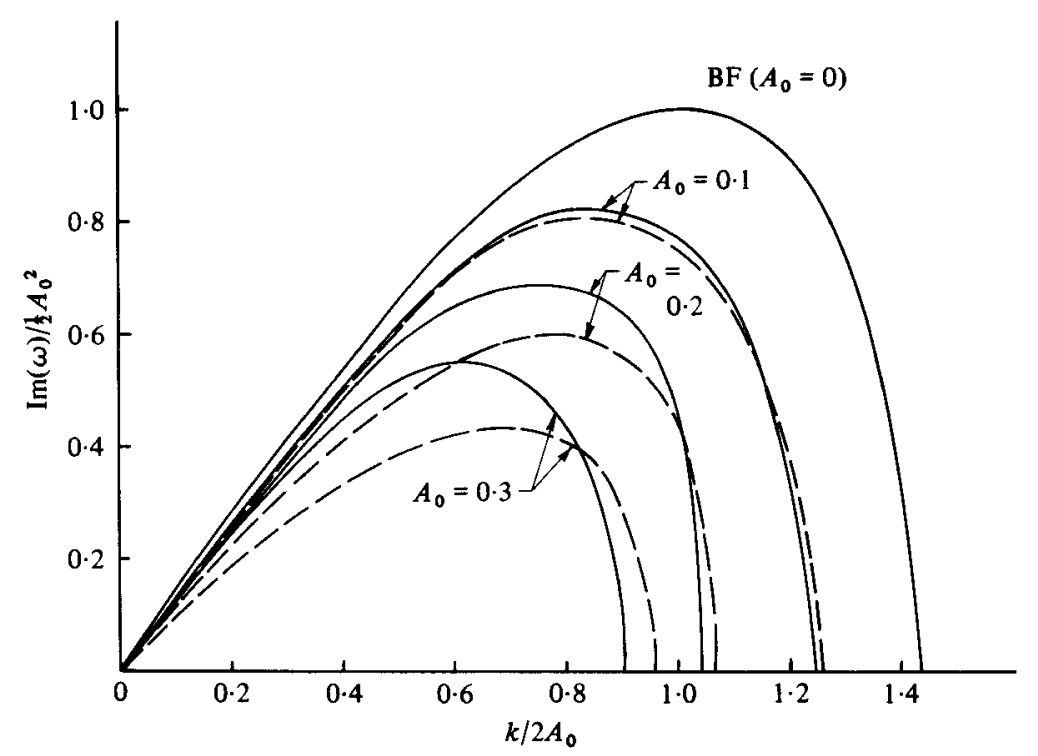

Figure 2. Sideband growth rate as a function of modulation wavenumber for various values of wave steepness $A_{0}$. - , (7); ---, results of Crawford et al. (1981).

\section{Recurrence}

In a previous paper (Janssen 1981) the long-time behaviour of the modulational instability (also called sideband instability or the Benjamin-Feir instability) was studied. By means of the multiple timescale method an approximate solution to the ordinary nonlinear Schrödinger equation was found, exhibiting recurrence (ef. the Fermi-Pasta-Ulam (1955) recurrence phenomenon). The result was in qualitative agreement with the numerical results of Yuen \& Ferguson (1978) and the experiment of Lake et al. (1977). Here we briefly consider the effect of the wave-induced current on the long-time behaviour of the modulational instability. We also discuss the generation of the frequency shift induced by the modulational instability, and we investigate whether there is a possible connection with the frequency shift found in the experiment of Lake et al. (1977).

In order to make a comparison with previous results (Janssen 1981) easier we introduce the scaling $t=4 \kappa^{2} t^{\prime}, x=\kappa x^{\prime}, A=A^{\prime} /(2 \kappa)^{\frac{1}{2}}$. Dropping the primes we may cast (4) in the form

$$
i \frac{\partial}{\partial t} A-\frac{1}{2} \frac{\partial^{2}}{\partial x^{2}} A=\kappa\left(|A|^{2}-\left|A_{0}\right|^{2}\right) A+A \mathscr{H}^{(1)} \frac{\partial}{\partial x}|A|^{2} .
$$

The dispersion relation (corresponding to (7)) for modulations of a uniform wavetrain reads in the new variables

$$
\omega^{2}=\frac{1}{4} k^{2}\left(k^{2}-4 A_{0}^{2}(\kappa-|k|)\right) .
$$

In order to investigate the effect of nonlinearity, we consider the initial-value problem for one particular modulation with a fixed wavenumber near the threshold for instability. The modulation is neutrally stable if the parameter $\kappa$ equals its critical value $\kappa_{\mathrm{c}}$ given by

$$
\kappa_{\mathrm{c}}=\frac{k^{2}}{4 A_{0}^{2}}+|k| \text {. }
$$


If $\kappa$ is increased above its critical value by a fractional amount $\Delta^{2}$,

$$
\kappa=\kappa_{\mathrm{c}}\left(1+\Delta^{2}\right),
$$

then the growth rate $\gamma \equiv|\operatorname{Im}(\omega)|$ of the modulation with wavenumber $k$ reads

$$
\gamma=\Delta k A_{0}\left(\kappa_{\mathrm{c}}\right)^{\frac{1}{2}}
$$

Hence a slight increase of the critical parameters of the order $\Delta^{2}$ already gives a growth rate of the modulation of the order $\Delta$. This singular behaviour near the threshold for instability, which is also illustrated by figures 1 and 2 , is typical for quadratic dispersion relations. It suggests the following 'expansion' of the time derivative:

$$
\frac{\partial}{\partial t}=\sum_{l=1}^{\infty} \Delta^{l} \frac{\partial}{\partial \tau_{l}}, \quad \tau_{l}=\Delta^{l} t
$$

where all $\tau_{l}$ are assumed to be independent of each other. In addition $A$ is expanded in powers of $\Delta$ :

$$
A=\sum_{l=0}^{\infty} \Delta^{l} A_{l}
$$

where the coefficients of expansion $A_{l}$ are functions of all $\tau_{l}$ and of $x$, except $A_{0}$, which is assumed to be a constant. Substitution of (1)), (13) and (14) into the fourth-order envelope equation (8) gives a hierarchy of equations of the form

$$
\mathscr{L} \boldsymbol{\Psi}_{l}=\mathbf{S}_{l} \quad(l=1,2,3, \ldots),
$$

where $\psi_{l}$ is a column vector with components $\operatorname{Re}\left(A_{l}\right)$ and $\operatorname{Im}\left(A_{l}\right), \mathscr{L}$ is a linear operator (i.e. $\mathscr{L} \boldsymbol{\Psi}_{l}=0$ is just the linear problem) and $\mathbf{S}_{l}$ is a source term containing only lower-order $\Psi_{p}(p \leqslant l-1)$.

We shall not give the details of the solution of (15) because they are similar to a case treated previously (Janssen (1981), where the ordinary nonlinear Schrödinger equation was treated in this fashion). We merely present the final results. To second order we obtain

where $A_{0}$ is real,

$$
A=\left(A_{0}+\Delta A_{1}+\Delta^{2} A_{2}\right) \exp i \sigma,
$$

$$
\begin{aligned}
A_{1} & =a \exp i k x+\text { c.c. } \\
A_{2} & =\frac{-|a|^{2}}{A_{0}}+2 \frac{\frac{3}{4} k^{2}-|k| A_{0}^{2}}{\frac{3}{4} k^{2}+|k| A_{0}^{2}}\left(\frac{a^{2}}{A_{0}} \exp 2 i k x+\text { c.c. }\right), \\
\sigma & =\sigma(0)-\frac{k^{2}}{A_{0}^{2}} \int_{0}^{\tau_{1}}|a|^{2} d \tau_{1}^{\prime}-\frac{2}{k^{2}}\left(\frac{\partial a}{\partial \tau_{1}} \exp i k x+\text { c.c. }\right) .
\end{aligned}
$$

Here the evolution in time of the complex amplitude $a$ follows from the requirement that the solutions of the inhomogeneous equation (15) be periodic in $x$-space (cf. Janssen 1981). The result is the following nonlinear evolution equation:

$$
\frac{\partial^{2}}{\partial r_{1}^{2}} a-\bar{\gamma}^{2} a+\beta^{2}|a|^{2} a=0,
$$

where $\bar{\gamma}=\kappa A_{0}\left(\kappa_{\mathrm{c}}\right)^{\frac{1}{2}}$, which is just the expression of the linear growth rate (cf. (12)), and +

$$
\beta^{2}=2 k^{2} \kappa_{\mathrm{c}}
$$

\footnotetext{
$\dagger$ In the expression for $\beta^{2}$ we have neglected terms of order $A_{0}^{4}$, consistent with a similar neglect of terms in the growth rate.
} 
The effect of wave-induced current is represented by the terms with the modulus of $k$. Since in the derivation of the nonlinear Schrödinger equation it was assumed that the wave steepness $A_{0}$ is of the same order as the modulation wavenumber $k$, we see at once that the effect of the wave-induced current may be neglected if $|k| \ll 1$. In the latter limit we rediscover the previous results on the ordinary nonlinear Schrödinger equation (Janssen 1981). As a final check, we note that (8) has a number of conserved quantities, e.g.

$$
\begin{gathered}
\frac{d}{d t} \int|\mathrm{A}|^{2} d x=0 \\
\frac{d}{d t} \int\left(A A_{x}^{*}-A^{*} A_{x}\right) d x=0 \\
\frac{d}{d t} \int d x\left\{\left|A_{x}\right|^{2}-\kappa|A|^{4}-|A|^{2} \mathscr{H}^{(1)}\left(|A|_{x}^{2}\right)\right\}=0
\end{gathered}
$$

Here, the integration in $x$-space extends over one wavelength. To second order in $\Delta$, we have checked that the solution (16) does indeed satisfy these conservation laws.

It is well known that (17) gives periodic solutions for $\beta^{2}>0$, since it is just the equation of motion of a particle in a potential well $V(|a|)=-\frac{1}{2} \bar{\gamma}^{2}|a|^{2}+\frac{1}{4} \beta^{2}|a|^{4}$. Thus, although the modulation is linearly unstable for small initial amplitudes, nonlinear effects are stabilizing, giving a periodic motion in time, since no disssipative effects are incorporated. Actually, in this case the main stabilization of the unstable modulation stems from the modification of the equilibrium (i.e. the term $-|a|^{2} / A_{0}$ in the expression for $A_{2}$ of (16)).

Hence, we have shown that the long-time behaviour of the modulational instability exhibits recurrence, qualitatively in agreement with experiments of Lake et al. (1977). In addition, it was observed that the upper sidebands have more energy than the lower sidebands. This feature is also reflected by our solution. To see this, we remark that Lake et al. have given power spectra of the surface elevation $\eta$. To second order in the wave steepness $\epsilon$, it has been shown by Hasimoto \& Ono (1972) that the relation between $\eta$ and the complex amplitude $A$ of the velocity potential $\phi$ is given by

$$
\eta=i \frac{k_{0}}{\omega_{0}}\left(A-\frac{i}{2 k_{0}} \frac{\partial}{\partial x} A\right) e^{i \theta}+\text { c.c. }
$$

which, because of the $\partial A / \partial x$ term, clearly illustrates the asymmetry between the lower and the upper sidebands.

However, in the experiment of Lake et al. also a down-shift in the frequency of the carrier wave was observed. Before we calculate this down-shift in the frequency, we note that this experiment refers to a boundary-value problem, instead of the initial-value problem considered in this paper. Therefore, the down-shift in the frequency of the experiment of Lake et al. corresponds to a shift in the wavenumber of the carrier wave in the initial-value problem. This wavenumber shift $\delta k_{0}$ may be obtained from the phase $\sigma$ of $A$ (cf. (16)):

$$
\delta k_{0}=\frac{\partial \sigma}{\partial x}=\frac{4}{k} \frac{\partial}{\partial \tau_{1}}(a) \sin k x
$$

where, for simplicity, we have assumed that $a$ is real. The point to be made now is that $\delta k_{0}$ is periodic in space and time, in contrast with the findings of Lake et al. (1977). For example, for the initial conditions $a(0)=a_{0}, \partial a(0) / \partial \tau_{1}=0$ we obtain from (17) 
that the solution returns to the initial conditions after a time $T$ (the recurrence time). This is of course the very nature of recurrence. Hence after a time $T$ the shift in wavenumber vanishes, which is certainly not the case in the experiment of Lake et al.

Thus the $x$-component of the wave-induced current (i.e. $\partial \bar{\phi} / \partial x$, giving the Hilbert-transform term in (8)) gives rise to a Doppler shift in e.g. the frequency of the carrier wave. However, this Doppler shift is periodic in time and space, in contrast with experimental findings.

We therefore conclude that other effects, not included in the present treatment, might explain the observed downshift of the frequency. An obvious candidate seems to be dissipation, although the nature of the dissipation process is unclear at the moment.

We only applied a simplified version of the fourth-order envelope equation to the stability of a finite-amplitude uniform wavetrain. We have checked, however, that the neglected terms only contribute to order $A_{0}^{4}$ in the evolution equation for the complex amplitude $a$ (cf. (17a)), i.e. they are of the same order as the neglected terms in the growth rate $\bar{\gamma}^{2}$. We are therefore justified in applying the simplified version of the fourth-order envelope equation to the nonlinear stability of a finite-amplitude uniform wavetrain.

\section{Nonlinear energy transfer in a narrow wave spectrum}

The previous sections were concerned with a deterministic field of gravity waves. We investigated the stability of a uniform wavetrain and studied the long-time behaviour of a simplified version of the fourth-order envelope equation. Let us now return to the fourth-order envelope equation (2) and let us consider the problem of energy transfer due to nonlinear interactions for a random homogeneous field of gravity waves.

This problem has been given much attention in recent years, and it was Hasselmann (1962) who obtained the evolution equation for the action density from a general perturbation theory. Longuet-Higgins (1976) derived the narrow-band limit of Hasselmann's equation by starting from the ordinary nonlinear Schrödinger equation. Dungey \& Hui (1979) pointed out, however, that the effect of spectral width for a typical wind-wave field on the nonlinear energy transfer is far from negligible. They were able to show this by expanding Hasselmann's coupling coefficient as a power series in a small parameter (which is a measure of the spectral width), and retaining only the first-order term. In the limit of vanishing spectral width the result of Longuet-Higgins was rediscovered.

In this section it is shown that the equation for the action which follows from the fourth-order envelope equation (2) is just the equation for the action density as derived by Dungey \& Hui (1979). Actually, one should expect this, and we shall show that the higher-order effects in the fourth-order envelope equation correspond to the first-order effect of spectral width in the equation for the nonlinear energy transfer of Dungey \& Hui (1979).

In order to see this we write $A$ as

$$
A=\int d \mathbf{k} a(\mathbf{k}, t) e^{i(\mathbf{k} \cdot \mathbf{x}-\mathbf{\Omega} t)}
$$

where $\Omega=\omega-\omega_{0}$ obeys the dispersion relation

$$
\Omega=\frac{1}{2} \lambda-\frac{1}{8} \lambda^{2}+\frac{1}{4} \mu^{2}-\frac{1}{16} \lambda^{3}-\frac{3}{8} \lambda \mu^{2} .
$$


Here, $\mathbf{k}=(\lambda, \mu)$ and $\omega_{0}=\left(g k_{0}\right)^{\frac{1}{2}}$ is the frequency of the carrier wave with wavenumber $k_{0}$. Substituting (21) into (2) we obtain after some algebra the following equation for the amplitude $a$ :

$$
\frac{\partial}{\partial t} a_{1}=-\frac{1}{2} i \int d \mathbf{k}_{2} d \mathbf{k}_{3} d \mathbf{k}_{4} D_{1,2,3,4} a_{2}^{*} a_{3} a_{4} \delta\left(\mathbf{k}_{1}+\mathbf{k}_{2}-\mathbf{k}_{3}-\mathbf{k}_{4}\right) \exp i\left(\omega_{1}+\omega_{2}-\omega_{3}-\omega_{4}\right) t
$$

where

$$
D_{1,2,3,4}=\left[1+\frac{1}{2} \lambda_{2}+3 \lambda_{3}-\frac{\left(\lambda_{2}-\lambda_{3}\right)^{2}}{\left[\left(\lambda_{2}-\lambda_{3}\right)^{2}+\left(\mu_{2}-\mu_{3}\right)\right]^{\frac{1}{2}}}\right]
$$

Here we have introduced the shorthand notation $a_{i}=a\left(\mathbf{k}_{i}\right)$ and have replaced $\Omega$ by $\omega$ in the exponent, because they differ only by a constant factor. We next return to dimensional units by observing that in (23) the time is made dimensionless by means of the frequency $\omega_{0}$ and the wavenumber $\mathbf{k}$ is made dimensionless by means of the wavenumber $k_{0}$. Using (19), we write

$$
a=k_{0}^{3} b\left(\frac{2 \omega_{0}}{g}\right)^{\frac{1}{2}}\left(1-\frac{\lambda}{4 k_{0}}\right),
$$

where $b b^{*}$ is just the action density. The result is

$$
\frac{\partial}{\partial t} b_{1}=-i \int d \mathbf{k}_{2} d \mathbf{k}_{3} d \mathbf{k}_{4} T_{1,2,3,4} b_{2}^{*} b_{3} b_{4}\left(\mathbf{k}_{1}+\mathbf{k}_{2}-\mathbf{k}_{3}-\mathbf{k}_{\mathbf{4}}\right) \exp i\left(\omega_{1}+\omega_{2}-\omega_{3}-\omega_{4}\right) t
$$

where

$$
\begin{array}{r}
T_{1,2,3,4}=k_{0}^{3}\left\{1+\frac{1}{k_{0}}\left[\frac{3}{2}\left(\lambda_{1}+\lambda_{2}\right)-\frac{1}{2} \frac{\left(\lambda_{2}-\lambda_{3}\right)^{2}}{\left[\left(\lambda_{2}-\lambda_{3}\right)^{2}+\left(\mu_{2}-\mu_{3}\right)^{2}\right]^{\frac{1}{2}}}\right.\right. \\
\left.\left.-\frac{1}{2} \frac{\left(\lambda_{1}-\lambda_{3}\right)^{2}}{\left[\left(\lambda_{1}-\lambda_{3}\right)^{2}+\left(\mu_{1}-\mu_{3}\right)^{2}\right]^{\frac{1}{2}}}\right]\right\} .
\end{array}
$$

In passing we note that the ordinary nonlinear Schrödinger equation would only give the factor 1 in the expression for $T_{1,2,3,4}$, so that the remaining terms stem from the higher-order effects in the fourth-order envelope equation.

The next step is to obtain an evolution equation for the action density $n=\left\langle b b^{*}\right\rangle$, where the angle brackets denote an ensemble average. The procedure to obtain the evolution of the action density is well-known (ef. Hasselmann 1962; Davidson 1969, 1972; Longuet-Higgins 1976; Crawford et al. 1981), so we omit the details.

The eventual result is

$$
\begin{aligned}
\frac{\partial}{\partial t} n_{1}=\int d \mathbf{k}_{2} d \mathbf{k}_{3} d \mathbf{k}_{4} G_{1,2,3,4} \delta\left(\mathbf{k}_{1}+\mathbf{k}_{2}-\mathbf{k}_{3}-\mathbf{k}_{4}\right) \\
\times \delta\left(\omega_{1}+\omega_{2}-\omega_{3}-\omega_{4}\right)\left[n_{3} n_{4}\left(n_{1}+n_{2}\right)-n_{1} n_{2}\left(n_{3}+n_{4}\right)\right],
\end{aligned}
$$

where $G_{1,2,3,4}=4 \pi T_{1,2,3,4}^{2}$. Hence, to first order in the spectral width, measured by $k / k_{0}$, we obtain

$$
\begin{gathered}
G_{1,2,3,4}=4 \pi k_{0}^{6}\left\{1+\frac{1}{k_{0}}\left[3\left(\lambda_{1}+\lambda_{2}\right)-\frac{\left(\lambda_{2}-\lambda_{3}\right)^{2}}{\left[\left(\lambda_{2}-\lambda_{3}\right)^{2}+\left(\mu_{2}-\mu_{3}\right)^{2}\right]^{\frac{1}{2}}}\right.\right. \\
\left.\left.-\frac{\left(\lambda_{1}-\lambda_{3}\right)^{2}}{\left[\left(\lambda_{1}-\lambda_{3}\right)^{2}+\left(\mu_{1}-\mu_{3}\right)^{2}\right]^{\frac{1}{2}}}\right]\right\} .
\end{gathered}
$$

The same expression was obtained by Dungey \& Hui (1979), expanding Webb's (1978) version of the coupling coefficient of Hasselmann (1962) in powers of the spectral width. In the limit of vanishing spectral width (corresponding to the neglect of the 
right-hand side of (2)) we rediscover the result of Longuet-Higgins (1976). In this limit, for spectra that are symmetrical with respect to the carrier wavenumber $k_{0}$, the resulting energy transfer is symmetrical (Dungey \& Hui 1979). The terms representing the effect of finite spectral width can be divided in two groups. The terms involving the square root, which arise from the Hilbert-transform term in (2), also give a symmetrical energy transfer if the spectrum is symmetrical, whereas the term $3\left(\lambda_{1}+\lambda_{2}\right) / k_{0}$ (arising from the remaining higher-order terms in (2)) gives an asymmetrical energy transfer. We should emphasize that Dungey \& Hui (1979) have shown numerically that the effect of finite spectral width is considerable, just as in the deterministic case treated in $\$ \S 2$ and 3 the effect of wave-induced current is far from negligible. Using the simple coupling coefficient (27), these authors obtained a close agreement with the Jonswap calculations (Hasselmann et al. (1973); they used the full expression for $G$ ) on the forward face of the spectrum.

We are therefore inclined to conclude that the fourth-order envelope equation (4) is a good starting point for the study of nonlinear effects in surface gravity waves.

\section{Conclusions}

The ordinary nonlinear Schrödinger equation for deep-water waves found by a perturbation analysis to $O\left(\epsilon^{3}\right)$ in the wave steepness $\epsilon$ compares unfavourably with the exact calculations of Longuet-Higgins, the results of Crawford et al. (1981) (who started from the Zakharov equations) and experimental results of Benjamin \& Feir (1967) and Lake et al. (1977). Dysthe (1979) achieved a considerable improvement by taking the perturbation analysis one step further to $O\left(\epsilon^{4}\right)$. The result is a coupled set of evolution equations for the envelope of the carrier wave and the wave-induced mean flow. From this set of equations we obtain a single evolution equation for the envelope, called the fourth-order envelope equation. The fourth-order effects give a surprising improvement compared with the ordinary nonlinear Schrödinger equation in many respects. Regarding the stability of a finite-amplitude uniform wavetrain the dominant new effect is the mean-flow response to non-uniformities in the radiation stress. For a wave steepness $\epsilon<0 \cdot 25$, the growth rate of the Benjamin-Feir instability is in good agreement with results of Crawford et al. (1981) and experiment (Benjamin \& Feir 1967; Lake et al. 1977). Also, a weakly nonlinear calculation shows that the amplitude of an unstable mode near the threshold for instability is periodic in time, therefore exhibiting recurrence. However, the observed irreversible downshift is not found from the fourth-order envelope equation, possibly because of the absence of dissipation. Finally, for a random homogeneous field of gravity waves, we derived the nonlinear energy transfer function, which agrees with the results of Dungey \& Hui (1979). The effect of finite spectral width as found by Dungey \& Hui corresponds to the $O\left(\epsilon^{4}\right)$ terms of the fourth-order envelope equation. For a symmetrical spectrum these terms give rise to an asymmetrical energy transfer. It is perhaps of interest to note that Roskes (1977) (although in his equation the Hilbert-transform term is missing) found these fourth-order terms to have a symmetry-breaking effect on the evolution of a single soliton, i.e. the soliton splits up into a large pulse followed by a small one.

We therefore conclude that the fourth-order envelope equation seems to be a good starting point for the study of nonlinear sea waves, since they usually have a wave steepness which is small enough (i.e. $\epsilon<0 \cdot 25$ ).

The author is pleased to acknowledge useful discussions with M. J. MeGuinness, P. G. Saffman and G. B. Whitham. 


\section{REFERENCES}

Benjamin, T. B. 1967 Instability of periodic wave trains in nonlinear dispersive systems. Proc. R. Soc. Lond. A 299, 59-75.

Benjamin, T. B. \& FeIr, J. E. 1967 The disintegration of wave trains on deep water. Part 1. Theory. J. Fluid Mech. 27, 417-430.

Crawford, D. R., Lake, B. M., Saffman, P. G. \& Yuen, H. C. 1981 Stability of weakly nonlinear deep-water waves in two and three dimensions. J. Fluid Mech. 105, 177-191.

Davidson, R. C. 1969 General weak turbulence theory of resonant four-wave processes. Phys. Fluids 12, 149-161.

Davidson, R. C. 1972 Methods in Nonlinear Plasma Theory. Academic.

Dungey, J. C. \& Hui, W. H. 1979 Nonlinear energy transfer in a narrow gravity-wave spectrum. Proc. R. Soc. Lond. A 368, 239-265.

Dysthe, K. B. 1979 Note on a modification to the nonlinear Schrödinger equation for application to deep water waves. Proc. $R$. Soc. Lond. A 369, 105-114.

Fermi, E., Pasta, J. \& Ulam, S. 1955 Studies of nonlinear problems. Los Alamos Rep. LA-1940 (May 1955) [In Collected Papers of Enrico Fermi, vol. 2, pp. 978-988. University of Chicago Press, 1962.]

Hasimoto, H. \& Ono, H. 1972 Nonlinear modulation of gravity waves. J. Phys. Soc. Japan 33, 805.

Hasselmann, K. 1962 On the nonlinear energy transfer in a gravity-wave spectrum. Part 1. General Theory. J. Fluid Mech. 12, 481.

Hasselmann, K., Barnett, T. P., Bouws, E., Carlson, H., Cartwright, D. E., Enke, K., Ewing, J. A., Gienapp, H., Hasselmann, D. E., Kruseman, P., Meerburg, A., Müller, P., Olbers, D.J., Richter, K., Seli, W. \& Walden, H. 1973 Measurements of wind-wave growth and swell decay during the Joint North Sea Wave Project (Jonswap), Deutsche Hydrogr. Z., Suppl. A (80), No. 12.

JAnssen, P. A. E. M. 1981 Modulational instability and the Fermi-Pasta-Ulam recurrence. Phys. Fluids 24, 23-26.

Lake, B. M., Yuen, H. C., Rungaldier, H. \& Ferguson, W. E. 1977. Nonlinear deep-water waves: theory and experiment. Part 2. Evolution of a continuous wave train. J. Fluid Mech. 83, 49-74.

LongUet-Higgins, M. S. 1976 On the nonlinear transfer of energy in the peak of a gravity-wave spectrum: a simplified model. Proc. R. Soc. Lond. A 347, 311-328.

LongUeT-Higgins, M. S. 1978 The instabilities of gravity waves of finite amplitude in deep water. II. Subharmonics. Proc. R. Soc. Lond. A 360, 489-505.

Longuet-Higgins, M. S. \& Stewart, R. W. 1964 Radiation stresses in water waves; a physical discussion, with applications. Deep-Sea Res. 11, 529-562.

Roskes, G. J. 1977 Fourth order envelope equation for nonlinear dispersive gravity waves. Phys. Fluids 20, 1576-1577.

WebB, D. J. 1978 Non-linear transfers between sea waves. Deep-Sea Res. 25, 279-298.

Yuen, H.C. \& Ferguson, W. E. 1978 Relationship between Benjamin-Feir instability and recurrence in the nonlinear Schrödinger equation. Phys. Fluids 21, 1275-1278.

ZAKHAROV, V. E. 1968 Stability of periodic waves of finite amplitude on the surface of a deep fluid. Zh. Prikl. Mekh. Tekh. Fiz. 9, 86-94. (Translated in J. Appl. Mech. Tech. Phys. 9, 190-194.) 